\title{
Long-Range Anomalous Decay of the Correlation in Jammed Packings
}

\author{
Paolo Rissone $\oplus^{1,{ }^{*}}$ Eric I. Corwin $\odot,{ }^{2, \dagger}$ and Giorgio Parisi $\oplus^{3,4,5, \dagger}$ \\ ${ }^{1}$ Small Biosystems Lab, Department of Condensed Matter Physics, Carrer de Marti i Franques, 1, 11, 08028 Barcelona, Spain \\ ${ }^{2}$ Department of Physics and Materials Science Institute, University of Oregon, Eugene, Oregon 97403, USA \\ ${ }^{3}$ Dipartimento di Fisica, Sapienza Università di Roma, P.le Aldo Moro 5, 00185 Rome, Italy \\ ${ }^{4}$ Istituto Nazionale di Fisica Nucleare, Sezione di Roma I, P.le A. Moro 5, 00185 Rome, Italy \\ ${ }^{5}$ Institute of Nanotechnology (NANOTEC)-CNR, Rome unit, P.le A. Moro 5, 00185 Rome, Italy
}

(Received 19 December 2020; revised 22 April 2021; accepted 5 May 2021; published 12 July 2021)

\begin{abstract}
We numerically study the structure of the interactions occurring in three-dimensional systems of hard spheres at jamming, focusing on the large-scale behavior. Given the fundamental role in the configuration of jammed packings, we analyze the propagation through the system of the weak forces and of the variation of the coordination number with respect to the isostaticity condition, $\Delta Z$. We show that these correlations can be successfully probed by introducing a correlation function weighted on the density-density fluctuations. The results of this analysis can be further improved by introducing a representation of the system based on the contact points between particles. In particular, we find evidence that the weak forces and the $\Delta Z$ fluctuations support the hypothesis of randomly jammed packings of spherical particles being hyperuniform by exhibiting an anomalous long-range decay. Moreover, we find that the large-scale structure of the density-density correlation exhibits a complex behavior due to the superimposition of two exponentially damped oscillating signals propagating with linearly depending frequencies.
\end{abstract}

DOI: 10.1103/PhysRevLett.127.038001

Introduction.-Amorphous packings of nearly incompressible particles, such as marbles and pebbles, have been the object of an intense investigation during the past decades as they represent a suitable benchmark for studying a broad range of dense-packing and optimization problems $[1,2]$. This rising interest led to the development of many experiments [3-5] and simulations [6-8] that made possible an extensive study of the features of these systems. Moreover, this field appeared to be the perfect environment to apply the theories of frustrated interactions [9]. In particular, the application of the replica theory [10] led to the elaboration of an exact analytical solution valid in the limit of high-dimensional packings [11-13].

We focus on athermal packings of frictionless hard spheres (HSs) compressed until particles come into mechanical contact with their nearest neighbors. The trapped spheres form a rigid network and cannot explore the surrounding environment (ergodicity breaking). Under these conditions, the system enters a phase of matter known as "jamming" $[14,15]$. It has been hypothesized that saturated jammed systems (no space to add another particle) are hyperuniform [16], implying that their radial distribution function (RDF) tends to zero from negative values as a power law [17] $g(r)-1 \propto r^{-4}$. Even though the tendency of jammed packings to hyperuniformity has been observed (with deviations from the postulated behavior) [18,19], such power-law scaling of the pair correlation function has never been directly measured.

In this framework, we find evidence of hyperuniformity in the long-range correlation of the forces -between adjacent particles and the deviation of the number of contacts per particle from the average value $\Delta Z=$ $Z-\langle Z\rangle$. On the one hand, jammed packings exhibit a unique force network [20] whose long-range fluctuations demand study. On the other hand, it has been shown that $\Delta Z$ exhibits interesting features at jamming [21-23] and that the fluctuations of the coordination number $\sigma_{Z}^{2}$ for a fixed $\Delta Z$ are similar to those of density hyperuniformity [24]. The research for a static observable exhibiting a nontrivial behavior close to jamming is motivated by the existence of a corresponding long-ranged dynamical response. It has been shown $[21,25,26]$ that a local perturbation to the position of a pair of adjacent particles, i.e., breaking the contact between particles, produces a response propagating through the system up to a maximum length, the "response length," $\xi_{R}$ that diverges at jamming [27]. The main hindrance to this analysis is represented by the strong statistical noise exhibited by the correlation functions in the long range and superimposing to the (weak) signal of interest. To overcome this problem, we define a suitable pair correlation function to point out the long-range behavior of the observables by filtering out the interfering signals. Moreover, we introduce a representation of the interparticle network based on the contact points between particles instead of their centers of mass. We show that the shift to a system of fictive particles improves the resolution of the correlation function and is fundamental in identifying the long-range features of the jammed packings. 
System Setup.-Given a system of $N$ randomly distributed monodisperse HSs of diameter $\sigma$, let us introduce the interaction via the dimensionless interaction potential

$$
U=\sum_{\langle i, j\rangle}\left(1-\frac{\left|\mathbf{r}_{i}-\mathbf{r}_{j}\right|}{\sigma_{i j}}\right),
$$

where $\mathbf{r}_{i, j}$ is the position of particles $i, j$ and $\sigma_{i j}$ is the distance between the centers of particles $i$ and $j$ when they are in contact. Note that, for monodisperse HSs, $\sigma_{i j}=\sigma$ and that, for particles in kissing contact, $r=\left|\mathbf{r}_{i}-\mathbf{r}_{j}\right|=\sigma$ so that the particles' interaction potential is zero.

The system is controlled via the "packing fraction" $\phi$, defined as the fraction of the system volume occupied by the spheres. The jammed phase is reached when the packing fraction hits the critical value $\phi_{J} \approx 0.64$ in three-dimensional systems [28-31]. By starting in the overjammed region $\left(\phi \approx 2 \phi_{J}\right), \phi$ is gradually decreased by gently shrinking the particles' diameter until the system reaches jamming. The jamming point is approached by iteratively minimizing the potential energy [Eq. (1)] using the FIRE algorithm [6] according to the protocol described in [20] (Appendix B). The simulation ends when the targeted precision is reached, i.e., when it is impossible to distinguish between a kissing contact and a small overlap.

At the end of this protocol, the particles form a network of enduring contacts that is stable only if the isostaticity condition holds [21,32-34], i.e., the average number of contacts per particle satisfies (in a first approximation) $\langle Z\rangle \equiv Z_{\text {iso }} \approx 2 d$, where $d$ is the system dimension. As shown in [35], this property depends on the system mechanical stability being ensured by the exact balancing between the affine and the nonaffine (negative) components of the interparticle interactions over the whole network. Within this picture, it is important to point out the existence of "bucklers," i.e., particles that still form part of the rigid network but that are minimally constrained so that $Z=$ $d+1$ [20]. A notable exception to the isostaticity condition is represented by "rattlers," particles that are not part of the contact network and freely move inside cages bounded by particles permanently in contact. The identification and the exclusion of rattlers are fundamental for obtaining reliable results. By adopting this method, we generated 96 critically jammed packings of $N=16384$ particles in $d=3$.

Generalized $R D F$.-Given a generic observable $\mathcal{O}$, we defined the "generalized pair correlation function" as

$$
C_{\mathcal{O}}^{s}(r)=\frac{g_{\mathcal{O}}^{s}(r)}{g(r)},
$$

where $r$ is the distance between particle pairs, $g(r)$ is the usual RDF [36], and

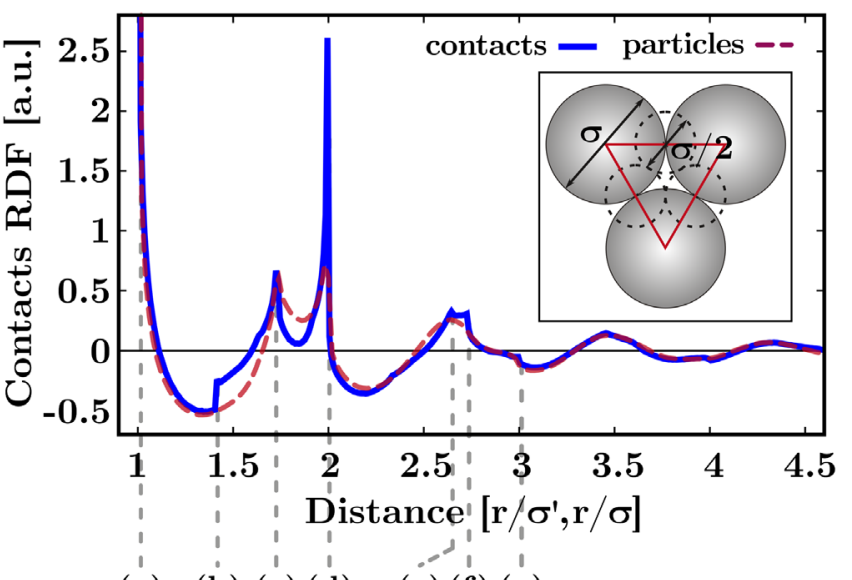

(a) (b) (c) (d)

(e) (f) (g) (e)

(c)

(a)

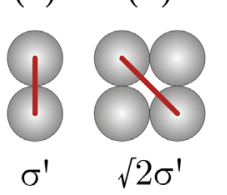

(b)

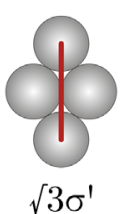

(d)

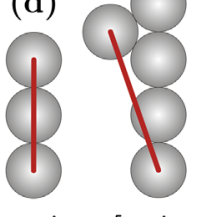

(f)

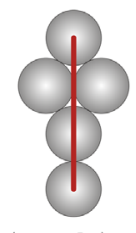

(g)

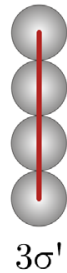

FIG. 1. Contacts and original RDFs (in blue and red, respectively) in $d=3$ as a function of the distance (expressed in the respective diameter units $r / \sigma^{\prime}$ and $r / \sigma$ ). Each peak (labeled with a different letter) occurs in correspondence to a different configuration of the fictive particles, as shown in the correspondent representations (which, however, do not exhaust all the possibilities), along with the distance at which each discontinuity appears (red lines). Inset: Schematic representation of the contacts-centered spheres model in $d=3$. Each fictive particle (dashed black lines) results from the contact of two HSs and has a diameter $\sigma^{\prime}=\sigma / 2$, being $\sigma$ the diameter of the original HS.

$$
g_{\mathcal{O}}^{s}(r)=\frac{1}{C} \sum_{i, j} \delta\left(\left|\mathbf{r}_{i}-\mathbf{r}_{j}\right|-r\right) \mathcal{O}_{i}^{s} \mathcal{O}_{j}^{s},
$$

$\mathbf{r}_{i}$ and $\mathbf{r}_{j}$ being the positions of particles $i$ and $j, C$ the normalization factor, and $s \in \Re$ a control parameter. Notice that by choosing $s=0$ in Eq. (3), one gets the RDF (additional details can be found in the Supplemental Material [37]). We studied the two cases $\mathcal{O}=f$ and $\mathcal{O}=\Delta Z \equiv Z-Z_{\text {iso }}, f$ being the force exchanged between particles and $\Delta Z$ the deviation of the number of contacts per particle from isostaticity.

To study the long-range correlations with higher accuracy, we also introduced the jammed packings' representation with respect to the contact points between particles instead of the centers of mass. To do this, let us consider the densest packing in which HSs can arrange (see the inset in Fig. 1). Each contact point between the HSs can be seen as the center of a fictive particle with radius $\sigma^{\prime}=\sigma / 2$. Thus, given a system of $N$ particles and $Z_{\text {iso }}=2 d$ average contacts per particle, the new contacts-based system will be formed by $N^{\prime} \leq 2 d N=6 N$ particles. 


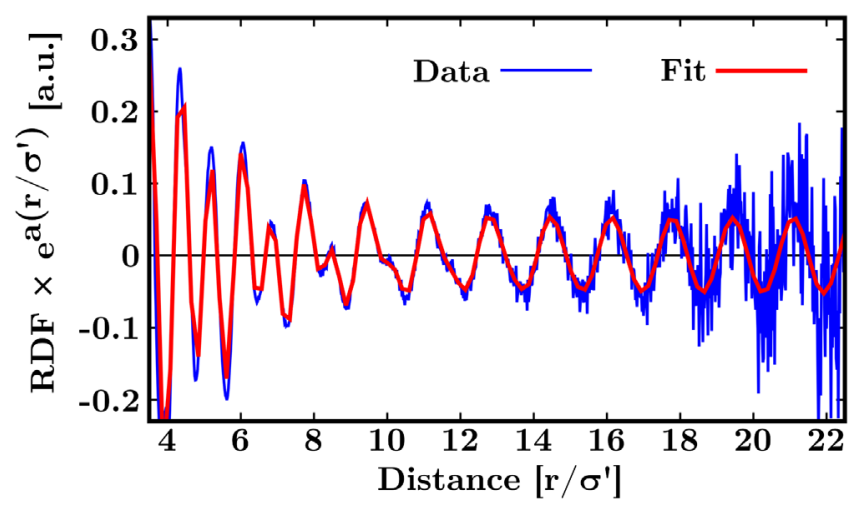

FIG. 2. Contacts density-density correlation $g\left(r / \sigma^{\prime}\right)$ multiplied by the factor $e^{a r / \sigma^{\prime}}$ with $a \approx 0.3$. Two sets of distinct oscillations given by Eq. (4) appear to be superimposed. The first one, $f_{m}(r)$, propagating in the middle range $[4: 9] r / \sigma^{\prime}$ and the second, $f_{l}(r)$, in the long-range region $[9: 22] r / \sigma^{\prime}$. By fitting the resulting function (red line), we found that $p_{l} \approx 7.5$ and $p_{m} \approx 3.8$. The distance is expressed in diameter units.

Contacts RDF.-Switching from the centers-of-massbased to the contact-points description of the network allowed us to study the radial distribution function of the contact-centered model (called "contacts RDF" in what follows) with a much higher resolution. Figure 1 shows the short-range behavior of the contacts RDF (blue line) compared to the real spheres RDF (red dashed line). The contacts RDF points out new features of the pair correlation function at jamming, evidencing discontinuities that were much smoother [peaks (e),(f),(g)] or completely absent [peak (b)] in the original system description. The higher accuracy of the contacts RDF is further reflected by the enhanced sharpness of peaks (c) and (d). The origin of each one of these discontinuities can be easily addressed. The first peak $\left(r=\sigma^{\prime}\right)$ is due to a nearest-neighbor contact, while the others are determined by different possible configurations of HSs forming a chain of contacts. Notably, peaks (c) and (d) in Fig. 1 correspond to "real" $\delta$ functions, i.e., to mechanically rigid configurations of perfectly centrosymmetric particles with respect to the transmission of forces. The local centrosymmetry ensures a zero nonaffine component of the response and therefore the full mechanical stability [38]. All the other peaks are the result of a wide range of possible arrangements. Interestingly, as shown in Fig. 2, the mid-range and long-range behavior of the correlation function appears to be exponentially damped as $e^{-a r}$, with $a \approx 0.3$. The enhancement of such behavior by considering $g(r) \times e^{a r}$ pointed out a superposition of two oscillatory functions of type

$$
f_{i}(r)=c_{i} e^{a_{i} r} \cos \left(p_{i} r+\psi_{i}\right),
$$

where $c_{i}, a_{i}, p_{i}, \psi_{i}$ are the function parameters and $i=m, l$ denotes the mid- and long-range regions, respectively. By performing the resulting eight parameters fit [37] $g(r)=f_{m}(r)+f_{l}(r)$, we found $p_{m} \approx 2 p_{l}=7.514 \pm$ 0.003 and $a_{m} \approx 2 a_{l}=0.70 \pm 0.01$. This result proves that, because the contribution of the long-range oscillations is small compared to the mid-range ones, the $f_{l}(r)$ can be considered as $\mathcal{O}(2)$ correction to the $\mathcal{O}(1)$ mid-range leading term $f_{m}(r)$.

Weak forces correlation.-At the jamming point, each particle of the system gets trapped in a fixed position by mechanical contact with its nearest neighbors. The stability of the resulting configuration is ensured by the balance of all the forces exchanged in these contact points, determining the formation of a complex forces network spreading through the whole system $[5,39,40]$. Within this picture, it is possible to distinguish between "strong" forces, which form a backbone crossing the whole system, and "weak" forces spreading only in small subregions of the system confined by branches of the main network (see the Supplemental Material [37]). Let us consider Eq. (3) with $\mathcal{O}=f$. By choosing $s<0$, the resulting $g_{f}^{s}(r)$ will be "weighted" on the weak forces so that the smaller the force, the bigger its contribution to the correlation. Note that not any value of $s$ can be chosen. In fact, as shown in [20], the force distribution can be described as function $P(f) \propto f^{\theta}$, with $\theta \approx 0.4$, Thus, the average force (raised to the power $s$ ) can be estimated as

$$
\left\langle f^{s}\right\rangle \sim \int d f f^{s} P(f)=\int d f f^{s} f^{\theta} \propto \frac{1}{1+\theta+s},
$$

which diverges unless $s>s_{\min } \equiv-1-\theta \approx-1.4$. Figure 3 shows the weak forces correlation $C_{f}^{s}(r)$ for $s=-1$ and $s=-1 / 2$. In both cases, the local density-density oscillations appear to be not completely damped, propagating up to large length scales and masking any power-law decay. Therefore, we introduced the correlation function averaged over the period $T$ of the short-range oscillations $\left\langle C_{f}^{s}(r)\right\rangle_{T}$ (orange circles), filtering out most of the additional periodic component. The period $T$ is roughly equal to the particle diameter but is tuned for each dataset. By fitting in the midand long-range region $[7: 23] r / \sigma^{\prime}$ and the resulting correlation functions to the power law $f(r)=A r^{-\gamma_{f}^{s}}+C$ for $s=$ $(-1,-1 / 2)$ (red line in the insets) with $C \approx 0$, we respectively found $\gamma_{f}^{s=-1}=0.7 \pm 0.3$ and $\gamma_{f}^{s=-1 / 2}=4.1 \pm 0.3$. These results prove that the fluctuations of the weak force correlation function at large length scales decay with the power law expected by the hyperuniformity theory, with the only constraint being a fine tuning of the selected (weak) forces. In fact, for $s=-1$, the fluctuations rapidly go to zero in the short range, whereas for $s=-1 / 2$ they exhibit a long-ranged anomalous decay with the measured nontrivial exponent.

Contacts correlation.- It has been proven that, at jamming, the coordination number per particle $Z$ plays an 

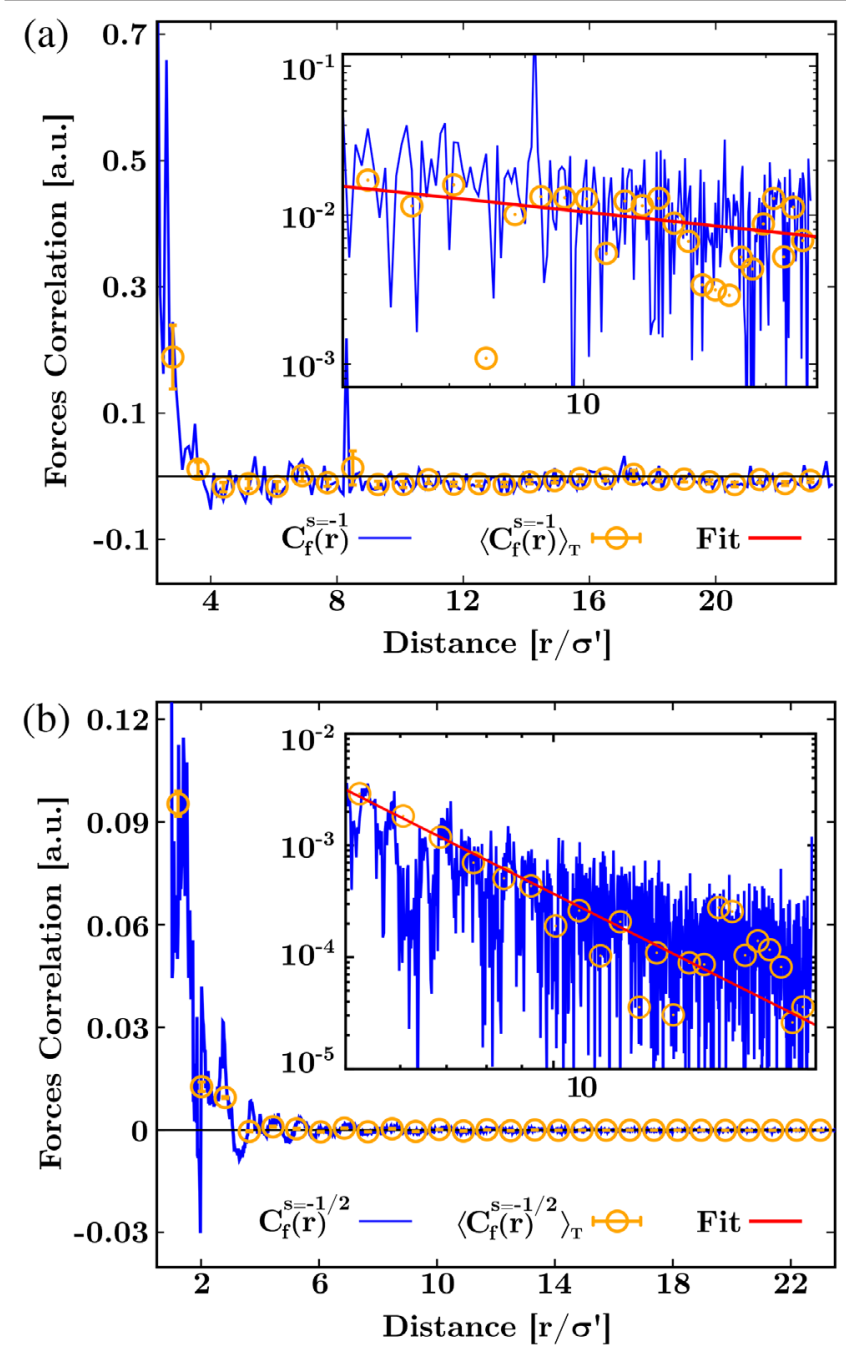

FIG. 3. (a) Weak force correlation function $C_{f}^{s=-1}(r)$ in $d=3$ and enlargement of the long-range region in log-log scale (inset). The density-density correlations propagating at long range have been filtered by introducing the average over the oscillation period $T,\left\langle C_{f}^{s}(r)\right\rangle_{T}$ (orange circles). The log-log scale in the inset points out a long-range power-law behavior (red line) decaying with an exponent $\gamma_{f}^{s=-1}=0.7 \pm 0.3$ in the range [7:23]. (b) Weak forces correlation function $C_{f}^{s=-1 / 2}(r)$ in $d=3$. The fluctuations analysis points out a power-law decay with an exponent $\gamma_{f}^{s=-1 / 2}=4.1 \pm 0.3$ in the range [7:23]. Notice that both insets show the modulus of the correlation function. The distances are expressed in diameter units.

essential role in determining the system features [24]. The study of the fluctuations $\sigma_{\Delta Z}^{2}$ led to the definition of a structural relaxation length $\xi_{\Delta Z} \propto \Delta Z^{-\nu}$, where $\Delta Z=Z-2 d, d$ is the system dimension, and $\nu$ is a nontrivial exponent. Here we point out that the $\Delta Z$ correlation also exhibits the expected anomalous behavior.

As previously discussed, changing to a contacts-based system representation also implies changing from a system of $N$ particles to one of $N^{\prime}=2 d N$ fictive particles. In this

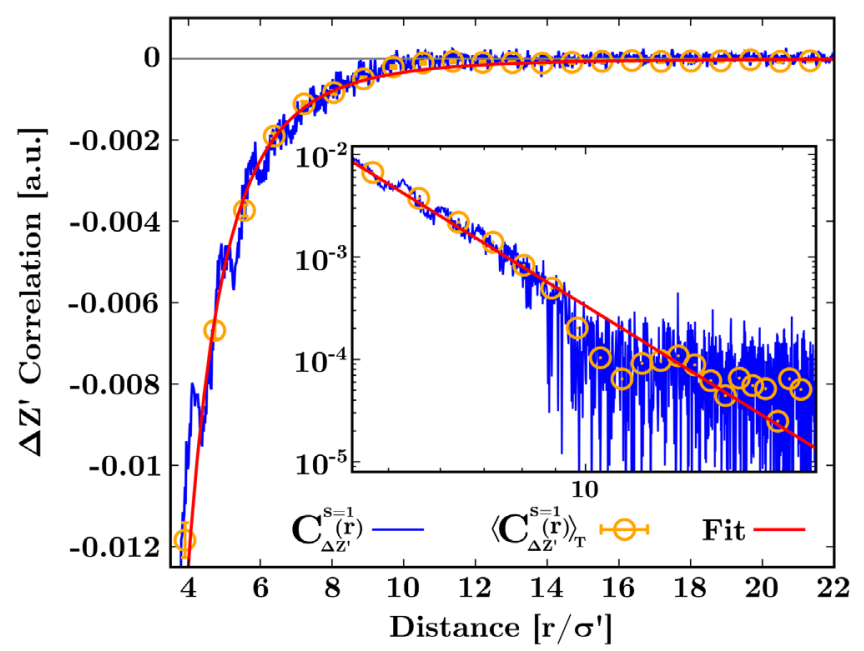

FIG. 4. Contact correlation function $C_{\Delta Z^{\prime}}^{s=1}(r)$ in $d=3$. The density-density oscillations at short range (blue line) have been filtered by introducing the correlation averaged over the oscillation period $T,\left\langle C_{\Delta Z^{\prime}}^{s=1}(r)\right\rangle$ (orange circles). Inset: Enlargement of the midand long-range regions of the modulus of the $C_{\Delta Z^{\prime}}^{s=1}(r)$ (blue line) in $\log -\log$ scale. The correlation function exhibits a power-law decay (red line) propagating with a nontrivial exponent $\gamma_{\Delta Z^{\prime}}=3.9 \pm 0.2$. The distance is expressed in diameter units.

framework, we defined the number of contacts per (fictive) particle as

$$
Z_{l}^{\prime}=\frac{Z_{i}+Z_{j}}{2}
$$

where $l=\left(1, \ldots, N^{\prime}\right)$ and $i, j=(1, \ldots, N)$ with $i \neq j$. Thus, we chose $\mathcal{O}=\Delta Z^{\prime}=Z^{\prime}-2 d$ and $s=1$ in Eqs. (3) and (2). The resulting correlation function $C_{\Delta Z^{\prime}}^{s=1}(r)$ (shown in Fig. 4) clearly exhibits a nontrivial behavior in the middle and long range superimposed onto some oscillations as a result of the incomplete damping of the density-density correlations. As described above, this power-low trend can be seen by fitting the period $T$ of the density-density oscillations and eventually recomputing Eq. (3) by considering spherical shells of thickness $T$ (orange circles in Fig. 4). Analogously to the previous case, we fit the resulting function according to the power law $f(r)=A r^{-\gamma_{\Delta Z^{\prime}}}+C$ in the range [4:23], finding a nontrivial exponent $\gamma_{\Delta Z^{\prime}}=3.9 \pm 0.2$.

Conclusion.-We studied the mid- and large-scale spatial correlations in HS packings at jamming by defining the correlation functions [Eqs. (3) and (2)] for the weak forces $f$ and the variation of the coordination number per particle $\Delta Z^{\prime}$. We found that the correlation functions exhibit a longrange anomalous behavior, supporting the hyperuniformity hypothesis and marking the transition to the jammed phase. In fact, both the weak forces and the $\Delta Z^{\prime}$ correlation exhibit a power-law decay with nontrivial exponents $\gamma_{f}^{s=-1} \approx \gamma_{\Delta Z^{\prime}} \approx 4$. Moreover, we introduced a representation of the system based on the particles' contact points instead 
of their centers of mass. This change pointed out new features of the density-density correlation function, which appears to be a sum of two different oscillating signals propagating in the middle and long range. A theoretical explanation for this two-terms form could be recovered by writing integral equations of the hypernetted chain kind [41] for the correlation function. However this problem has not been explored yet. It would be of great interest to obtain such proof. Changing to the contacts-based representation of the system also increased the sensitivity of the correlation function at long range.

These results show that static observables other than the density can be used to characterize the main features of jammed systems, marking (expected) theoretical behaviors that remained hidden by studying the density fluctuations of the pair correlation function. A further step in this analysis would involve the study of bigger systems, i.e., systems with a larger number of particles $N$, implying easier isolation of the power-law decay with respect to the local oscillations. Moreover, it would be of great interest to characterize these correlation functions at a packing fraction $\phi<\phi_{J}$ close to jamming and varying it up to the jamming point.

This work was supported by a Simons Foundation grant (No. 454939, EC; No. 454949, GP).

Note added-During the production of our work, we became aware of [19].

*Corresponding author.

paolo.rissone@ub.edu

Corresponding author.

ecorwin@uoregon.edu

*Corresponding author.

giorgio.parisi@roma1.infn.it

[1] S. Boettcher and M. Grigni, Jamming model for the extremal optimization heuristic, J. Phys. A 35, 1109 (2002).

[2] F. Krzakala and J. Kurchan, Landscape analysis of constraint satisfaction problems, Phys. Rev. E 76, 021122 (2007).

[3] T. Majmudar, M. Sperl, S. Luding, and R. P. Behringer, Jamming Transition in Granular Systems, Phys. Rev. Lett. 98, 058001 (2007).

[4] J. Zhang, T. Majmudar, M. Sperl, and R. Behringer, Jamming for a 2d granular material, Soft Matter 6, 2982 (2010).

[5] R. Behringer, D. Bi, B. Chakraborty, A. Clark, J. Dijksman, J. Ren, and J. Zhang, Statistical properties of granular materials near jamming, J. Stat. Mech. (2014) P06004.

[6] E. Bitzek, P. Koskinen, F. Gähler, M. Moseler, and P. Gumbsch, Structural Relaxation Made Simple, Phys. Rev. Lett. 97, 170201 (2006).

[7] C. Zhang, C. B. O’Donovan, E. I. Corwin, F. Cardinaux, T. G. Mason, M. E. Möbius, and F. Scheffold, Structure of marginally jammed polydisperse packings of frictionless spheres, Phys. Rev. E 91, 032302 (2015).
[8] E. I. Corwin, M. Clusel, A. O. Siemens, and J. Brujić, Model for random packing of polydisperse frictionless spheres, Soft Matter 6, 2949 (2010).

[9] B. Charbonneau, P. Charbonneau, and G. Tarjus, Geometrical frustration and static correlations in hard-sphere glass formers, J. Chem. Phys. 138, 12A515 (2013).

[10] G. Parisi and F. Zamponi, A replica approach to glassy hard spheres, J. Stat. Mech. (2009) P03026.

[11] J. Kurchan, G. Parisi, and F. Zamponi, Exact theory of dense amorphous hard spheres in high dimension I. The free energy, J. Stat. Mech. (2012) P10012.

[12] J. Kurchan, G. Parisi, P. Urbani, and F. Zamponi, Exact theory of dense amorphous hard spheres in high dimension. II. The high density regime and the gardner transition, J. Phys. Chem. B 117, 12979 (2013).

[13] P. Charbonneau, J. Kurchan, G. Parisi, P. Urbani, and F. Zamponi, Exact theory of dense amorphous hard spheres in high dimension. III. The full replica symmetry breaking solution, J. Stat. Mech. (2014) P10009.

[14] G. Biroli, Jamming: A new kind of phase transition? Nat. Phys. 3, 222 (2007)

[15] P. Charbonneau, J. Kurchan, G. Parisi, P. Urbani, and F. Zamponi, Glass and jamming transitions: From exact results to finite-dimensional descriptions, Annu. Rev. Condens. Matter Phys. 8, 265 (2016).

[16] S. Torquato and F. H. Stillinger, Local density fluctuations, hyperuniformity, and order metrics, Phys. Rev. E 68, 041113 (2003).

[17] A. Donev, S. Torquato, and F. Stillinger, Pair correlation function characteristics of nearly jammed disordered and ordered hard-sphere packings, Phys. Rev. E 71, 011105 (2005)

[18] A. Ikeda, L. Berthier, and G. Parisi, Large-scale structure of randomly jammed spheres, Phys. Rev. E 052125 (2017).

[19] S. Wilken, R. E. Guerra, D. Levine, and P. M. Chaikin, following Letter, Random Close Packing as a Dynamical Phase Transition, Phys. Rev. Lett. 127, 038002 (2021).

[20] P. Charbonneau, E. Corwin, G. Parisi, and F. Zamponi, Jamming Criticality Revealed by Removing Localized Buckling Excitations, Phys. Rev. Lett. 114, 125504 (2015).

[21] M. Wyart, L. Silbert, S. Nagel, and T. Witten, Effects of compression on the vibrational modes of marginally jammed solids, Phys. Rev. E 72, 051306 (2005).

[22] N. C. Karayiannis, K. Foteinopoulou, and M. Laso, Contact network in nearly jammed disordered packings of hardsphere chains, Phys. Rev. E 80, 011307 (2009).

[23] C. F. Moukarzel, Elastic collapse in disordered isostatic networks, Europhys. Lett. 97, 36008 (2012).

[24] D. Hexner, A. Liu, and S. Nagel, A diverging length scale in the structure of jammed systems, in In APS March Meeting Abstracts (2018), Vol. 2018, pp. C47-013.

[25] E. Degiuli, E. Lerner, and M. Wyart, Theory of the jamming transition at finite temperature, J. Chem. Phys. 142, 164503 (2015).

[26] M. Müller and M. Wyart, Marginal stability in structural, spin, and electron glasses, Annu. Rev. Condens. Matter Phys. 6, 177 (2015).

[27] A. J. Liu and S. R. Nagel, The jamming transition and the marginally jammed solid, Annu. Rev. Condens. Matter Phys. 1, 347 (2010). 
[28] A. Donev, S. Torquato, and F. H. Stillinger, Neighbor list collision-driven molecular dynamics simulation for nonspherical hard particles. I. Algorithmic details, J. Comput. Phys. 202, 737 (2005).

[29] K. Kim and T. Munakata, Glass transition of hard sphere systems: Molecular dynamics and density functional theory, Phys. Rev. E 68, 021502 (2003).

[30] L. E. Silbert, A. J. Liu, and S. R. Nagel, Structural signatures of the unjamming transition at zero temperature, Phys. Rev. E 73, 041304 (2006).

[31] E. Somfai, M. van Hecke, W. G. Ellenbroek, K. Shundyak, and W. van Saarloos, Critical and noncritical jamming of frictional grains, Phys. Rev. E 75, 020301 (2007).

[32] M. Van Hecke, Jamming of soft particles: Geometry, mechanics, scaling and isostaticity, J. Phys. Condens. Matter 22, 033101 (2009).

[33] S. Torquato and F. H. Stillinger, Jammed hard-particle packings: From kepler to bernal and beyond, Rev. Mod. Phys. 2633 (2010).

[34] C. Coulais, Dense vibrated granular media: From stuck liquids to soft solids, $\mathrm{PhD}$ thesis, Université Pierre et Marie Curie-Paris VI, 2012.
[35] A. Zaccone and E. Scossa-Romano, Approximate analytical description of the nonaffine response of amorphous solids, Phys. Rev. E 184205 (2011).

[36] D. Frenkel and B. Smit, Understanding Molecular Simulation: From Algorithms to Applications (Academic Press, New York, 2001).

[37] See Supplemental Material at http://link.aps.org/ supplemental/10.1103/PhysRevLett.127.038001 for a detailed description of the generalized RDF, the eight parameters fit and the origin of the weak forces.

[38] R. Milkus and A. Zaccone, Local inversion-symmetry breaking controls the boson peak in glasses and crystals, Phys. Rev. B 93, 094204 (2016).

[39] D. Wang, J. Ren, J. A. Dijksman, H. Zheng, and R. P. Behringer, Microscopic Origins of Shear Jamming for 2d Frictional Grains, Phys. Rev. Lett. 120, 208004 (2018).

[40] R. Hartley and R. Behringer, Logarithmic rate dependence of force networks in sheared granular materials, Nature (London) 421, 928 (2003).

[41] J.-P. Hansen and I. McDonald, Theory of Simple Liquids (Elsevier, New York, 1990). 